\title{
Model Hamiltonians in Density Functional Theory
}

\author{
Paola Gori-Giorgi, Julien Toulouse, and Andreas Savin
}

\begin{abstract}
The formalism of Kohn and Sham uses a specific (model) Hamiltonian which highly simplifies the many-electron problem to that of noninteracting fermions. The theorem of Hohenberg and Kohn tells us that, for a given ground state density, this Hamiltonian is unique. In principle, this density can be chosen as that of the real, interacting system. To obtain the energy, or other properties of the real system, approximations are needed. Working with non interacting fermions is an important simplification, but it may be easier to produce approximations with different choices of the model Hamiltonian. The feature that the exact density is (ideally) reproduced can be kept in the newly defined fictitious systems. Using model Hamiltonians having the same form as the physical one, that is, being built of one- and two-body operators, allows to approach the physical Hamiltonian arbitrarily close, and thus a systematic reduction of the approximations.
\end{abstract}

\section{Introduction}

1.1. General context. The knowledge from first principles of the electronic structure of atoms, molecules and solids is contained in the $N$-electron Schrödinger equation that, within the Born-Oppenheimer approximation (i.e., at fixed nuclei positions $\boldsymbol{R}_{\alpha}$ ), reads

$$
\widehat{H}\left(\mathbf{r}_{1}, \ldots, \mathbf{r}_{N}\right) \Psi\left(\mathbf{r}_{1} \sigma_{1}, \ldots, \mathbf{r}_{N} \sigma_{N}\right)=E \Psi\left(\mathbf{r}_{1} \sigma_{1}, \ldots, \mathbf{r}_{N} \sigma_{N}\right),
$$

with

$$
\begin{gathered}
\widehat{H}=\widehat{T}+\widehat{W}_{e e}+\widehat{V}_{n e}, \\
\widehat{T}=-\frac{1}{2} \sum_{i=1}^{N} \nabla_{\mathbf{r}_{i}}^{2}, \\
\widehat{W}_{e e}=\frac{1}{2} \sum_{i \neq j}^{N} \frac{1}{\left|\mathbf{r}_{i}-\mathbf{r}_{j}\right|} \equiv \frac{1}{2} \sum_{i \neq j}^{N} w_{e e}\left(\left|\mathbf{r}_{i}-\mathbf{r}_{j}\right|\right), \\
\widehat{V}_{n e}=-\sum_{i=1}^{N}\left(\sum_{\alpha=1}^{M} \frac{Z_{\alpha}}{\left|\boldsymbol{R}_{\alpha}-\mathbf{r}_{i}\right|}\right) \equiv \sum_{i=1}^{N} v_{n e}\left(\mathbf{r}_{i}\right),
\end{gathered}
$$

2000 Mathematics Subject Classification. Primary: 81-08; Secondary: 81Q05.

This is the final form of the paper. 
where $v_{n e}$ is the external potential due to the $M$ nuclei of charges $Z_{\alpha}$ at positions $\boldsymbol{R}_{\alpha}$, and Hartree atomic units, $\hbar=1$ (reduced Planck's constant) $m=1$ (electron mass), $a_{0}=1$ (Bohr radius), $e=1$ (electron charge), have been used. Since electrons obey the Fermi-Dirac statistics, $\Psi$ must be antisymmetric under particle exchange, $\Psi\left(\mathbf{r}_{1} \sigma_{1}, \ldots, \mathbf{r}_{i} \sigma_{i}, \ldots, \mathbf{r}_{j} \sigma_{j}, \ldots, \mathbf{r}_{N} \sigma_{N}\right)=-\Psi\left(\mathbf{r}_{1} \sigma_{1}, \ldots, \mathbf{r}_{j} \sigma_{j}, \ldots, \mathbf{r}_{i} \sigma_{i}, \ldots\right.$, $\left.\mathbf{r}_{N} \sigma_{N}\right)$, where $\mathbf{r}$ denotes the three-dimensional electronic position and $\sigma$ the spin degree of freedom $(\uparrow$ or $\downarrow)$. In what follows we will be only concerned with the search for the ground-state energy, i.e., the lowest eigenvalue $E_{0}$ of (1.1).

The methods that both chemists and physicists have developed to find approximate solutions of (1.1) can be roughly divided in two large groups: wavefunction methods (traditional quantum chemistry methods [28], quantum Monte Carlo [7]) and density methods (density functional theory [18], and density matrix functional theory [4], that is somehow in between the two groups). Simplistically, wave-function methods start from an approximation for the antisymmetric, normalized, $N$-electron wave-function, $\Psi_{\text {approx }}$, and take advantage of the variational principle,

$$
E_{0} \leq E_{0}^{\text {approx }}=\min _{\Psi_{\text {approx }}}\left\langle\Psi_{\text {approx }}|\widehat{H}| \Psi_{\text {approx }}\right\rangle .
$$

Since $\Psi_{\text {approx }}$ is a $3 N$-dimensional object, wave-function methods are in general computationally expensive when the number of particles increases.

Density Functional Theory (DFT) uses the electron density $n(\mathbf{r})$ as a basic variable,

$$
n(\mathbf{r})=N \sum_{\sigma_{1}, \ldots, \sigma_{N}} \int\left|\Psi\left(\mathbf{r} \sigma_{1}, \mathbf{r}_{2} \sigma_{2}, \ldots, \mathbf{r}_{N} \sigma_{N}\right)\right|^{2} d \mathbf{r}_{2}, \ldots, d \mathbf{r}_{N},
$$

a much simpler quantity to handle, resulting in a low computational cost that allows to reach system sizes much larger than those accessible to wave-function methods. At given number of electrons $N$, the Hohenberg and Kohn theorem [17] tells us that the ground-state density $n(\mathbf{r})$ of (1.7) completely determines (except for an additive constant) the external potential $v_{n e}(\mathbf{r})$ of (1.5) (for the sake of simplicity we only consider physical Hamiltonians with a nondegenerate ground state). Since the kinetic energy operator of (1.3) and the electron-electron interaction of (1.4) are the same for all systems, a universal functional $F$ of the density $n(\mathbf{r})$ can be defined as [22]

$$
F\left[n ; \widehat{W}_{e e}, \widehat{T}\right]=\min _{\Psi \rightarrow n}\left\langle\Psi\left|\widehat{T}+\widehat{W}_{e e}\right| \Psi\right\rangle,
$$

where, to keep the connection with what we will do in the next sections, we have explicitly shown the dependence of $F$ on the electronic interaction $\widehat{W}_{e e}$ and on the kinetic energy operator $\widehat{T}$. The minimum search in (1.8) is performed over all antisymmetric wave-functions $\Psi$ that yield the density $n(\mathbf{r})$ (by definition $n(\mathbf{r})$ also gives, by integration, the number of electrons $N)$. The universal functional $F$ can be also defined as a Legendre transform [23]

$$
F\left[n ; \widehat{W}_{e e}, \widehat{T}\right]=\sup _{v}\left\{\min _{\Psi}\left\langle\Psi\left|\widehat{T}+\widehat{W}_{e e}+\widehat{V}\right| \Psi\right\rangle-\int n(\mathbf{r}) v(\mathbf{r}) d \mathbf{r}\right\},
$$

where, as in the rest of this paper, $\widehat{V}$ denotes a local one-body operator of the form of (1.5) with $v_{n e}(\mathbf{r})$ replaced by $v(\mathbf{r})$. If the exact form of the functional $F$ was 
known, the variational principle would tell us that

$$
E_{0}=\min _{n}\left\{F\left[n ; \widehat{W}_{e e}, \widehat{T}\right]+\int n(\mathbf{r}) v_{n e}(\mathbf{r}) d \mathbf{r}\right\} .
$$

In practice, because we rely on approximations for $F$, the energy estimated by carrying out the minimization in (1.10) can be lower than the exact $E_{0}$.

1.2. Kohn-Sham density functional theory. The Kohn-Sham [19] approach to DFT introduces another density functional $T_{s}[n]$,

$$
T_{s}[n] \equiv F[n ; 0, \widehat{T}]=\sup _{v}\left\{\min _{\Phi}\langle\Phi|\widehat{T}+\widehat{V}| \Phi\rangle-\int n(\mathbf{r}) v(\mathbf{r}) d \mathbf{r}\right\},
$$

where, as in the rest of this work, $\Phi$ always denotes the wave-function of a spin$\frac{1}{2}$ fermionic system with zero electron-electron interaction, i.e., in the majority of cases, a single Slater determinant (the fundamental idea of Kohn-Sham has been to introduce the fermionic statistic in the construction of $T_{s}[n]$ ). Since dealing with noninteracting particles is computationally simple, Kohn and Sham [19] proposed to search for that particular noninteracting system which has the same ground-state density of the physical one. This defines a model system which is usually called Kohn-Sham (KS) system. The difference between $F\left[n ; \widehat{W}_{e e}, \widehat{T}\right]$ and $T_{s}[n]$ defines the Hartree-exchange-correlation functional $E_{\mathrm{Hxc}}[n]$,

$$
E_{\mathrm{Hxc}}[n]=F\left[n ; \widehat{W}_{e e}, \widehat{T}\right]-T_{s}[n]
$$

that needs to be approximated. The functional derivative, $\delta E_{\mathrm{Hxc}}[n] / \delta n(\mathbf{r})=$ $v_{\mathrm{Hxc}}(\mathbf{r})$, determines the one-body KS potential $v_{\mathrm{KS}}(\mathbf{r})=v_{n e}(\mathbf{r})+v_{\mathrm{Hxc}}(\mathbf{r})$ that forces the $N$ noninteracting electrons to have the same density of the physical system. The KS-DFT relies thus on the assumption that, given a physical ground-state density $n(\mathbf{r})$, it is possible to find a noninteracting system which has the same ground-state density.

From the functional $E_{\mathrm{Hxc}}[n]$ the classical electrostatic Hartree term is usually extracted,

$$
E_{\mathrm{H}}[n]=\frac{1}{2} \int d \mathbf{r} \int d \mathbf{r}^{\prime} \frac{n(\mathbf{r}) n\left(\mathbf{r}^{\prime}\right)}{\left|\mathbf{r}-\mathbf{r}^{\prime}\right|},
$$

and the remaining, unknown, part is called exchange-correlation energy,

$$
E_{\mathrm{xc}}[n]=F\left[n ; \widehat{W}_{e e}, \widehat{T}\right]-T_{s}[n]-E_{\mathrm{H}}[n] .
$$

The Hartree functional $E_{\mathrm{H}}[n]$ describes the electrons as if they were classical charge distributions. It is a simple functional of the density, and yields an important part of the energy of a many-electron system, but is nonzero also for a one-electron density. This "self-interaction part" of $E_{\mathrm{H}}[n]$ is cancelled by the exact $E_{\mathrm{xc}}[n]$, but this does not occur for most of the current approximate functionals, which suffer of the so called "self-interaction error".

The success of KS-DFT is mostly due to the fact that simple approximations (local-density approximation and generalized gradient corrections) for $E_{\mathrm{xc}}[n]$ and its functional derivative provide practical estimates of thermodynamical, structural and spectroscopic properties of atoms, molecules and solids. However, with the current approximations, KS-DFT is still lacking in several aspects, in particular it fails to handle near-degeneracy correlation effects (rearrangement of electrons within partially filled shells) and to recover long-range van der Waals interaction 
energies. The inaccuracy of KS-DFT stems from our lack of knowledge of $E_{\mathrm{xc}}[n]$, and much effort is put nowadays in finding new approximations to this term [24]. A trend in the current research is to construct implicit functionals of the density: in particular, the exchange-correlation functional is divided into exact exchange

$$
E_{x}[n]=\left\langle\Phi\left|\widehat{W}_{e e}\right| \Phi\right\rangle-E_{\mathrm{H}}[n],
$$

and the remaining correlation energy $E_{c}[n]=E_{\mathrm{xc}}[n]-E_{x}[n]$. The exact exchange cancels the self-interaction error of $E_{\mathrm{H}}[n]$, and is an implicit functional of $n(\mathbf{r})$ through the Slater determinant $\Phi$. The corresponding Kohn-Sham potential must be determined via the optimized effective potential method (OEP) [20,37],

$$
E_{0}=\inf _{v}\left\{\left\langle\Phi_{v}\left|\widehat{T}+\widehat{W}_{e e}+\widehat{V}_{n e}\right| \Phi_{v}\right\rangle+E_{c}\left[n_{\Phi_{v}}\right]\right\}
$$

where $\Phi_{v}$ is the ground state of the noninteracting Hamiltonian, $\widehat{T}+\widehat{V}$. The construction of a correlation energy functional $E_{c}[n]$ to be used with exact exchange in (1.16) is still an open problem.

In this work, we review some basic ideas, results, and open questions of a different approach: instead of trying to approximate the $\operatorname{KS~}_{\mathrm{xc}_{\mathrm{c}}}[n]$, we change the model system defined by (1.11).

1.3. Adiabatic connection formula. Before discussing the choice of different model Hamiltonians, we report some equations that will be used in the next sections. An exact formula for the functional $E_{\mathrm{Hxc}}[n]$ can be obtained via the adiabatic connection formalism $[30,36]$ : by varying a real parameter $\lambda$, the interaction $w^{\lambda}\left(r_{12}\right)$ between the electrons (we have defined $r_{12}=\left|\mathbf{r}_{1}-\mathbf{r}_{2}\right|$ to denote pairwise interactions that only depend on the electron-electron distance) is switched on continuously from zero to $1 / r_{12}$, while the density is kept fixed by an external one-body potential $\widehat{V}^{\lambda}$. Each Hamiltonian $\widehat{H}^{\lambda}$ along this adiabatic connection has a ground-state wavefunction $\Psi^{\lambda}$ that yields, by construction, the same density $n(\mathbf{r})$ for each $\lambda$. If $w^{\lambda=0}=0$ and $w^{\lambda=a}=1 / r_{12}$, the KS Hartree-exchange-correlation energy is given by $[30,36]$

$$
E_{\mathrm{Hxc}}[n]=\int_{0}^{a} d \lambda \int_{0}^{\infty} d r_{12} 4 \pi r_{12}^{2} f^{\lambda}\left(r_{12}\right) \frac{\partial w^{\lambda}\left(r_{12}\right)}{\partial \lambda},
$$

where the spherically and system-averaged pair density (APD) $f^{\lambda}\left(r_{12}\right)$ is obtained by integrating $\left|\Psi^{\lambda}\right|^{2}$ over all variables but $r_{12}=\left|\mathbf{r}_{2}-\mathbf{r}_{1}\right|$,

$$
\begin{aligned}
& f^{\lambda}\left(r_{12}\right) \\
& \quad=\frac{N(N-1)}{2} \sum_{\sigma_{1}, \ldots, \sigma_{N}} \int\left|\Psi^{\lambda}\left(\mathbf{r}_{12}, \boldsymbol{R}, \mathbf{r}_{3}, \ldots, \mathbf{r}_{N}\right)\right|^{2} \frac{d \Omega_{\mathbf{r}_{12}}}{4 \pi} d \boldsymbol{R} d \mathbf{r}_{3}, \ldots, d \mathbf{r}_{N} .
\end{aligned}
$$

where $\boldsymbol{R}=\left(\mathbf{r}_{1}+\mathbf{r}_{2}\right) / 2$.

\section{Changing the Model Hamiltonian}

2.1. General considerations. Equation (1.11) shows that the KS approach to DFT introduces a model Hamiltonian in which $\widehat{W}_{e e}$ is set equal to zero and the one-body potential, $v_{\mathrm{KS}}(\mathbf{r})$, is different from $v_{n e}(\mathbf{r})$ (and is obtained by imposing the condition that the ground-state electron density of the model Hamiltonian be the same of the physical system). Solving the noninteracting KS Hamiltonian instead of the fully interacting Hamiltonian of (1.1) is obviously very practical. The exact 

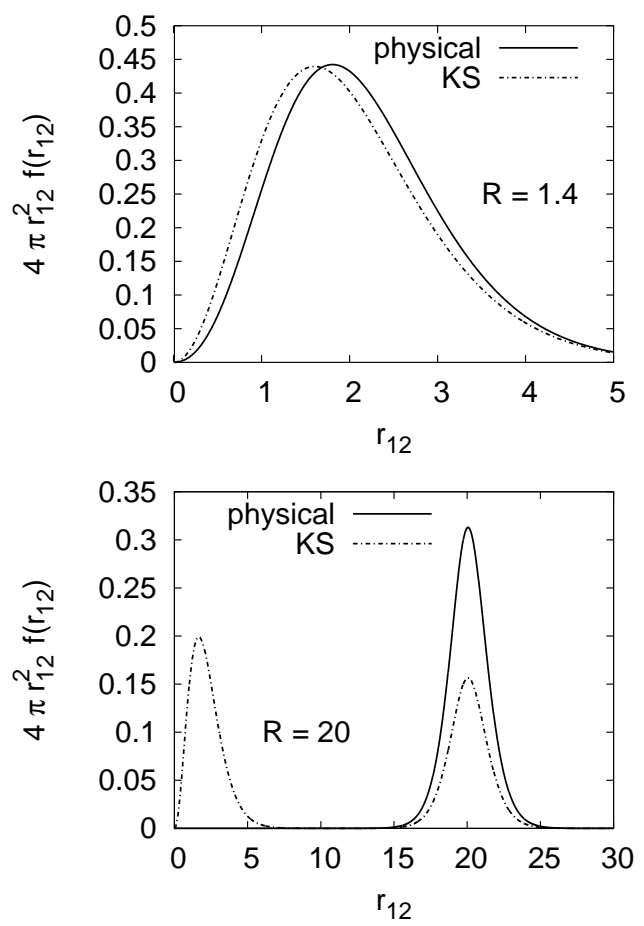

FIGURE 1. The spherically and system-averaged pair density $f^{\lambda}\left(r_{12}\right)$ of $(1.18)$ for the $\mathrm{H}_{2}$ molecule in the KS system $(\lambda=0)$ and in the physical system (Coulombic interaction). The internuclear equilibrium distance $R=1.4$ and the extreme stretched molecule at $R=20$ are considered.

ground-state energy of the physical system can, in principle, be obtained from the KS Slater determinant $\Phi$ via the functional $E_{\mathrm{xc}}[n]$. However, there are cases in which the restriction that the model system be noninteracting makes the search for an approximate $E_{\mathrm{xc}}[n]$ seem like a daunting task. Consider the simple example of the $\mathrm{H}_{2}$ molecule (two electrons in the field of two nuclei of unitary charge spaced by a distance $R$ ). In Figure 1 we report the quantity $4 \pi r_{12}^{2} f^{\lambda}\left(r_{12}\right)$ that enters in (1.17) for the KS system and for the physical system $(\lambda=0$ and $\lambda=a$, respectively) at two internuclear distances $R$. As shown by (1.17), the change in the APD $f^{\lambda}\left(r_{12}\right)$ when we switch from the KS system to the physical (fully interacting) system determines the functional $E_{\mathrm{Hxc}}[n]$. If this change is not drastic, we can expect that universal approximations for $E_{\mathrm{Hxc}}[n]$ can work relatively well. This is, e.g., the case of the $\mathrm{H}_{2}$ molecule at the equilibrium distance $R=1.4$ a.u., reported in Figure 1. But when we stretch the molecule (e.g., in the extreme case $R=20$ considered in the same figure), we see that the two APD are completely different, which means that the term $E_{\mathrm{Hxc}}[n]$ becomes very important: it has to correct the very different nature of the KS wavefunction with respect to the physical one. This effect is completely system-dependent and it is thus very difficult to include in a universal functional of the density. 
Cases like this appear when we have near-degenerate levels in the Kohn-Sham system (in the case of the $\mathrm{H}_{2}$ molecule described above, the energies of the two KS states $\sigma_{g}$ and $\sigma_{u}$ become closer and closer as $R$ increases). The basic idea reviewed in this paper is to remove the constraint that the model system be noninteracting in order to keep a reasonable resemblance between the model wavefuntion and the real one.

2.2. Using a modified electron-electron interaction. Obviously, the model system must still be less expensive to solve than the physical one. A possible approach is to define a density functional for a "partial" electron-electron interaction $w\left(r_{12}\right) \geq 0$, smaller than the Coulomb interaction $\forall r_{12}, w\left(r_{12}\right) \leq 1 / r_{12}$, but different from zero (we restrict our choice to pairwise interactions that only depend on the electron-electron distance). Following the idea of the adiabatic connection of Section 1.3, we can make our partial interaction depend on a real parameter $\mu$ in such a way that $w^{\mu}\left(r_{12}\right) \rightarrow 0$ when $\mu \rightarrow 0$ and $w^{\mu}\left(r_{12}\right) \rightarrow 1 / r_{12}$ when $\mu$ tends to some positive value $a$, and define

$$
F^{\mu}[n] \equiv F\left[n ; \widehat{W}^{\mu}, \widehat{T}\right]=\sup _{v}\left\{\min _{\Psi}\left\langle\Psi\left|\widehat{T}+\widehat{W}^{\mu}+\widehat{V}\right| \Psi\right\rangle-\int n(\mathbf{r}) v(\mathbf{r}) d \mathbf{r}\right\} .
$$

With this definition, we see that the density functional $F^{\mu}[n]$ switches from the Kohn-Sham one of (1.11) to the physical one of (1.9),

$$
\begin{aligned}
& F^{\mu \rightarrow 0}[n]=F[n ; 0, \widehat{T}]=T_{s}[n], \\
& F^{\mu \rightarrow a}[n]=F\left[n ; \widehat{W}_{e e}, \widehat{T}\right] .
\end{aligned}
$$

In analogy with the KS approach, we can ask that the model system with interaction $w^{\mu}\left(r_{12}\right)$ have the same density of the physical one. This fixes the external one-body potential (that we call $\left.v^{\mu}(\mathbf{r})\right)$ in our model Hamiltonian $\widehat{H}^{\mu}$. The groundstate wavefunction of our model system is denoted $\Psi^{\mu}$ : it is a multideterminantal wavefunction that must be computed with one of the standard methods of quantum chemistry (configuration interaction, coupled cluster, multi-configurational self-consistent field,...). In general, if $\mu$ is not too large (i.e., if $w^{\mu}$ is still much smaller than the full Coulomb interaction), few determinants describe $\Psi^{\mu}$ quite accurately, so that the computational cost can be kept low. The difference $\bar{F}^{\mu}[n]$ between the physical functional $F\left[n ; \widehat{W}_{e e}, \widehat{T}\right]$ and the partially-interacting $F^{\mu}[n]$,

$$
\bar{F}^{\mu}[n]=F\left[n ; \widehat{W}_{e e}, \widehat{T}\right]-F\left[n ; \widehat{W}^{\mu}, \widehat{T}\right],
$$

is what we need to approximate, together with its functional derivative that determines $v_{n e}(\mathbf{r})-v^{\mu}(\mathbf{r})$.

How to choose $w^{\mu}\left(r_{12}\right)$ ? Two points are important to determine the partial interaction: (i) that we can design reasonable approximations for the corresponding $\bar{F}^{\mu}[n]$, and (ii) that we can solve adequately the Hamiltonian $\widehat{H}^{\mu}$ of the model system. A convenient choice seems to be a long-ranged interaction, i.e., a $w^{\mu}\left(r_{12}\right)$ that behaves as $1 / r_{12}$ for large $r_{12}$, but that is softer than $1 / r_{12}$ for small $r_{12}$. The reasons for this choice are (i) short-range correlation effects seem to be more transferable from one system to another [2], and they should thus be more easily described in terms of an approximate universal density functional; (ii) the traditional wavefunction methods of quantum chemistry can reasonably describe $\Psi^{\mu}$ at a lower cost than the fully interacting system, because of the smaller interaction and because of the absence of the electron-electron cusp. For practical reasons (analytic 
matrix elements for both Gaussians and plane-waves, i.e. the most commonly used basis sets in quantum chemistry and solid-state physics, respectively), a common choice is

$$
w^{\mu}\left(r_{12}\right)=\frac{\operatorname{erf}\left(\mu r_{12}\right)}{r_{12}}
$$

where $\operatorname{erf}(x)$ is the error function (and we thus have $a=\infty$ ). Other possibilities for $w^{\mu}\left(r_{12}\right)$ have been also explored: they are all, like (2.5), arbitrary, and an open question is wether there is a way to determine $w^{\mu}\left(r_{12}\right)$ according to some optimal criteria. The approach resulting from the choice of $(2.5)$ is the one that we describe more in details in the next Section 3. Before doing that, for completeness we also briefly introduce a different choice of the model Hamiltonian.

2.3. Adding a nonlocal one-body operator. Another way to modify the model system is by adding a nonlocal one-body operator, $\widehat{O}_{\mathrm{NL}}$, multiplied by a real, positive constant $g[15,29]$,

$$
F^{g}[n] \equiv F\left[n ; \widehat{T}+g \widehat{O}_{\mathrm{NL}}, \widehat{W}_{e e}\right] .
$$

By setting $\widehat{O}_{\mathrm{NL}}$ equal to the projector (here reported for closed-shell systems) onto the virtual Kohn-Sham orbitals $\phi_{i}(\mathbf{r})$,

$$
\widehat{O}_{\mathrm{NL}}\left(\mathbf{r}, \mathbf{r}^{\prime}\right)=\delta\left(\mathbf{r}-\mathbf{r}^{\prime}\right)-\sum_{i=1}^{N / 2} \phi_{i}(\mathbf{r}) \phi_{i}^{*}\left(\mathbf{r}^{\prime}\right),
$$

the functional $F^{g}[n]$ of (2.6) switches from the physical one at $g=0$ to the KohnSham one of (1.11) in the limit $g \rightarrow \infty$ (since $\left\langle\Psi\left|\widehat{O}_{\mathrm{NL}}\right| \Psi\right\rangle$ is always $\geq 0$, as $g \rightarrow \infty$ the model system minimizes its energy by making $\left\langle\Psi\left|\widehat{O}_{\mathrm{NL}}\right| \Psi\right\rangle=0$, that is, by occupying only the KS orbitals). This choice of the model system is not further detailed here; the interested reader may find additional information in the literature $[6,15,29]$.

\section{Multideterminantal DFT from a Long-Range-Only Interaction}

3.1. The functionals. Following the same steps of Section 1.3, we can write an exact formula for the functional $\bar{F}^{\mu}[n]$ of $(2.4)$ in terms of the APD $f^{\mu}\left(r_{12}\right)$. It is convenient to use for the adiabatic connection the same partial interaction $w^{\mu}\left(r_{12}\right)$ of (2.5) chosen to determine the model system,

$$
\bar{F}^{\mu}[n]=\int_{\mu}^{\infty} d \mu^{\prime} \int_{0}^{\infty} d r_{12} 4 \pi r_{12}^{2} f^{\mu^{\prime}}\left(r_{12}\right) \frac{\partial w^{\mu^{\prime}}\left(r_{12}\right)}{\partial \mu^{\prime}} .
$$

This formula is identical to (2.4), except from the fact that the integration over the coupling constant starts from the positive value $\mu$ instead than zero.

As in KS-DFT, the functional $\bar{F}^{\mu}[n]$ can be divided into an Hartree term,

$$
\bar{E}_{\mathrm{H}}^{\mu}[n]=\frac{1}{2} \int d \mathbf{r} \int d \mathbf{r}^{\prime} n(\mathbf{r}) n\left(\mathbf{r}^{\prime}\right)\left[\frac{1}{\left|\mathbf{r}-\mathbf{r}^{\prime}\right|}-w^{\mu}\left(\left|\mathbf{r}-\mathbf{r}^{\prime}\right|\right)\right],
$$

and an exchange-correlation term

$$
\bar{E}_{\mathrm{xc}}^{\mu}[n]=\bar{F}^{\mu}[n]-\bar{E}_{\mathrm{H}}^{\mu}[n]
$$


that needs to be approximated. The functional $\bar{E}_{\mathrm{xc}}^{\mu}[n]$ can, in turn, be divided into exchange and correlation in two different ways. We can, in fact, define an exchange functional by using the Kohn-Sham determinant $\Phi$,

$$
\bar{E}_{\mathrm{x}}^{\mu}[n]=\left\langle\Phi\left|\widehat{W}_{e e}-\widehat{W}^{\mu}\right| \Phi\right\rangle-\bar{E}_{\mathrm{H}}^{\mu}[n],
$$

and then define the usual correlation energy functional $\bar{E}_{c}^{\mu}[n]$,

$$
\bar{E}_{c}^{\mu}[n]=\bar{E}_{\mathrm{xc}}^{\mu}[n]-\bar{E}_{\mathrm{x}}^{\mu}[n]
$$

but we can also define a multideterminantal (md) exchange functional [34] by using the wavefunction $\Psi^{\mu}$,

$$
\bar{E}_{\mathrm{x}, \mathrm{md}}^{\mu}[n]=\left\langle\Psi^{\mu}\left|\widehat{W}_{e e}-\widehat{W}^{\mu}\right| \Psi^{\mu}\right\rangle-\bar{E}_{\mathrm{H}}^{\mu}[n],
$$

and then a corresponding correlation energy,

$$
\bar{E}_{\mathrm{c}, \mathrm{md}}^{\mu}[n]=\bar{E}_{\mathrm{xc}}^{\mu}[n]-\bar{E}_{\mathrm{x}, \mathrm{md}}^{\mu}[n]
$$

These two ways of splitting exchange and correlation play a role only if we implement an optimized effective potential-like scheme, in analogy with what is usually done for the exact-exchange KS-DFT $[20,37]$ [see $(1.16)]$. In the case of the multideterminantal exchange of (3.6) the problem can be reformulated as [34]

$$
E_{0}=\inf _{v^{\mu}}\left\{\left\langle\Psi_{v^{\mu}}^{\mu}\left|\widehat{T}+\widehat{W}_{e e}+\widehat{V}_{n e}\right| \Psi_{v^{\mu}}^{\mu}\right\rangle+\bar{E}_{\mathrm{c}, \mathrm{md}}^{\mu}\left[n_{\Psi_{v^{\mu}}^{\mu}}\right]\right\},
$$

where $\Psi_{v^{\mu}}^{\mu}$ is obtained by solving the Schrödinger equation corresponding to the Hamiltonian $\widehat{H}^{\mu}=\widehat{T}+\widehat{W}^{\mu}+\widehat{V}^{\mu}$. This equation is the generalization of (1.16) to the case of the multideterminantal model system $\Psi^{\mu}$.

3.2. Exact properties of the functionals. From (3.1) it is possible to derive exact properties of the functionals of Eqs. (3.4)-(3.7) in the $\mu \rightarrow 0$ limit $[25,32]$ and in the $\mu \rightarrow \infty$ limit $[14,32]$.

In the first case, all the functionals $\bar{E}_{x}^{\mu}, \bar{E}_{c}^{\mu}$ [Eqs. (3.4) and (3.5)] and $\bar{E}_{\mathrm{x}, \mathrm{md}}^{\mu}$, $\bar{E}_{\mathrm{c}, \mathrm{md}}^{\mu}$ [Eqs. (3.6) and (3.7)] tend to the KS functionals of Section 1.2. This limit can be studied with perturbation theory: one finds that the way in which the functionals approach the KS ones depends on whether the system is confined [32] (atoms, molecules) or extended [25].

The large- $\mu$ limit is the most interesting, since, as shown by (3.1), it always lies in the range of $\mu$-values for which we want to construct approximations. In this limit, all the functionals of Eqs. (3.4)-(3.7) vanish and, since $\partial w^{\mu}\left(r_{12}\right) / \partial \mu=$ $2 / \sqrt{\pi} e^{-\mu^{2}} r_{12}^{2}$, equation (3.1) shows that their large- $\mu$ behavior is determined by the short-range part (small $r_{12}$ ) of the spherically and system-averaged pair density $f^{\mu}\left(r_{12}\right)$. It is possible to show [14] that, when $\mu \rightarrow \infty$, the small- $r_{12}$ part of $f^{\mu}\left(r_{12}\right)$ is dominated by

$$
f^{\mu}\left(r_{12}\right)=f(0)\left[1+2 r_{12} p_{1}\left(\mu r_{12}\right)+\frac{2}{\sqrt{\pi} \mu}\right]
$$

where $f(0)$ is the "on-top value" (zero electron-electron distance) of the physical (i.e., corresponding to the Coulomb interaction) $f\left(r_{12}\right)$, and the function $p_{1}(y)$ is equal to [14]

$$
p_{1}(y)=\frac{e^{-y^{2}}-2}{2 \sqrt{\pi} y}+\left(\frac{1}{2}+\frac{1}{4 y^{2}}\right) \operatorname{erf}(y)
$$


Inserting (3.9) (and, for the case of $E_{x}^{\mu}[n]$, the KS $f^{\mu=0}\left(r_{12}\right)$, corresponding to the KS determinant $\Phi)$ into (3.1) we find, for unpolarized systems $[8,14,32]$

$$
\begin{aligned}
& \bar{E}_{x}^{\mu \rightarrow \infty}[n]=-\frac{\pi}{4 \mu^{2}} \int d \mathbf{r} n(\mathbf{r})^{2}+O\left(\frac{1}{\mu^{4}}\right) \\
& \bar{E}_{c}^{\mu \rightarrow \infty}[n]=\frac{\pi}{\mu^{2}}\left[f(0)-\frac{1}{4} \int d \mathbf{r} n(\mathbf{r})^{2}\right]+f(0) \frac{4 \sqrt{2 \pi}}{3 \mu^{3}}+O\left(\frac{1}{\mu^{4}}\right) \\
& \bar{E}_{\mathrm{x}, \mathrm{md}}^{\mu \rightarrow \infty}[n]=\frac{\pi}{\mu^{2}}\left[f(0)-\frac{1}{2} \int d \mathbf{r}, n(\mathbf{r})^{2}\right]+f(0) \frac{4 \sqrt{\pi}(2 \sqrt{2}-1)}{3 \mu^{3}}+O\left(\frac{1}{\mu^{4}}\right) \\
& \bar{E}_{\mathrm{c}, \mathrm{md}}^{\mu \rightarrow \infty}[n]=-f(0) \frac{4 \sqrt{\pi}(\sqrt{2}-1)}{3 \mu^{3}}+O\left(\frac{1}{\mu^{4}}\right)
\end{aligned}
$$

These equations tell us that (i) if we use the definition of exchange with the KS determinant, the functional $\bar{E}_{x}^{\mu}[n]$ is, for large $\mu$, exactly described by a local functional of the density; (ii) all the other three functionals involve the physical "on-top" $f(0)$, which is usually not available (its knowledge would require a very accurate calculation for the physical system!). However, it is possible to construct approximations for $f(0)$. For instance, for many systems the estimate of $f(0)$ from the local density approximation (i.e., by transfer from the uniform electron gas model) is rather good [2]. Another possibility is to use (3.9) to estimate $f(0)$ from $f^{\mu}(0)$. This estimate has the advantage of being without self-interaction error [14].

3.3. Building approximate functionals. Following the same historical path of KS-DFT, the simplest approximation one can think of is the local density (LDA),

$$
\bar{E}_{\mathrm{xc}}^{\mu}[n]=\int d \mathbf{r} n(\mathbf{r})\left[\varepsilon_{\mathrm{xc}}(n(\mathbf{r}))-\varepsilon_{\mathrm{xc}}^{\mu}(n(\mathbf{r}))\right],
$$

where $\varepsilon_{\mathrm{xc}}(n)$ and $\varepsilon_{\mathrm{xc}}^{\mu}(n)$ are the exchange-correlation energy per particle of an electron gas of uniform density $n$ with interaction $1 / r_{12}$ [3] and $w^{\mu}\left(r_{12}\right)[25,35]$, respectively.

Generalized-gradient approximations (GGA) for $\bar{E}_{\mathrm{xc}}^{\mu}$ have been also constructed $[9,10,16,33]$ along similar lines of KS-DFT [26]. In this context, we only mention a conceptually simple approximation based on the exact properties of the previous Section 3.2. It consists [33] in a rational interpolation between a given GGA $\mathrm{KS}$ functional at $\mu=0$, and zero at $\mu \rightarrow \infty$. The rational interpolation is constrained to recover the exact large- $\mu$ behavior of Eqs. (3.11)-(3.12), using the LDA approximation for the physical on-top $f(0)$.

All these ways of constructing approximate functionals are based on the assumption of transferability of short-range exchange and correlation effects from one system (the uniform electron gas) to the others. Another strategy that came out recently, and that seems very well suited to describe short-range correlation effects, consists in generating realistic APD $f^{\mu^{\prime}}\left(r_{12}\right)$ along the adiabatic connection [with $\mu^{\prime} \geq \mu$, to be inserted in (3.1)] by solving simple "radial" (unidimensional) equations $[12,13]$ for a set of "effective geminals" $\psi_{i}^{\mu^{\prime}}\left(r_{12}\right)$,

$$
\begin{gathered}
{\left[-\frac{1}{r_{12}} \frac{d^{2}}{d r_{12}^{2}} r_{12}+\frac{\ell(\ell+1)}{r_{12}^{2}}+w_{\mathrm{eff}}^{\mu^{\prime}}\left(r_{12}\right)\right] \psi_{i}^{\mu^{\prime}}\left(r_{12}\right)=\varepsilon_{i}^{\mu^{\prime}} \psi_{i}^{\mu^{\prime}}\left(r_{12}\right)} \\
\sum_{i} \vartheta_{i}\left|\psi_{i}^{\mu^{\prime}}\left(r_{12}\right)\right|^{2}=f^{\mu^{\prime}}\left(r_{12}\right)
\end{gathered}
$$


The effective interaction $w_{\text {eff }}^{\mu^{\prime}}\left(r_{12}\right)$ is (in principle) the Lagrange multiplier for the exact $f^{\mu^{\prime}}\left(r_{12}\right)$ (i.e., corresponding to the wavefunction $\Psi^{\mu^{\prime}}$ along the adiabatic connection; in practice $w_{\text {eff }}^{\mu^{\prime}}\left(r_{12}\right)$ is approximated). In these equations the rule for the occupancy $\vartheta_{i}$ of the effective geminals is chosen, for spin compensated systems, to be Slater-determinant-like: occupancy 1 for even $\ell$ (singlet symmetry), occupancy 3 for odd $\ell$ (triplet symmetry), up to $N(N-1) / 2$ occupied geminals. This rule has been applied to solve the effective Eqs. (3.16) in the uniform electron gas at $\mu=\infty$ (Coulombic interaction), with rather accurate results $[5,11]$ when combined with simple approximations for the effective interaction potential $w_{\text {eff }}\left(r_{12}\right)$.

3.4. The calculation of $\Psi^{\mu}$ : an illustrative example. As said in the previous paragraphs, the model wavefunction $\Psi^{\mu}$ is, in most cases, computed with one of the traditional wavefunction methods of quantum chemistry. We report here an illustrative example of such calculation, and we then give in the next Section 4 an overview of recent results obtained using different methods for computing $\Psi^{\mu}$, combined with different approximations for the functionals.

Since the only purpose of this paragraph is to investigate the wavefunction part of the multideterminantal DFT, we only consider two very small systems, the He and the Be atoms, for which it has been possible to construct very accurate potentials $v^{\mu}[32]$. In this way (i) we have essentially no approximation on the functional part and we can focus our attention on the effect of approximations on the calculation of $\Psi^{\mu}$, and (ii) we can produce accurate benchmark results to test our calculations. Of course, this is not the general procedure in which we are finally interested. The general procedure rather consists in using a given approximation (see Section 3.3) for the functional $\bar{E}_{\mathrm{xc}}^{\mu}[n]$ and the corresponding potential, and combining it with a wavefunction method to calculate $\Psi^{\mu}$, to finally obtain the total ground state energy of the physical Hamiltonian. This procedure has been followed with very promising results in the works reviewed in the next Section 4.

Here, in order to illustrate the efficiency of approximate wavefunction methods to treat $\Psi^{\mu}$, we thus proceed as follows [32]. We first construct, for each $\mu$, the model Hamiltonian $\widehat{H}^{\mu}$ using an accurate potential $v^{\mu}$ [32] and compute accurately its ground-state energy, $E^{\mu}=\left\langle\Psi^{\mu}\left|\widehat{H}^{\mu}\right| \Psi^{\mu}\right\rangle$, at the multi-reference configuration interaction with singles and doubles (MRCISD) level. We then compute various approximate ground-state energies, $E_{S}^{\mu}=\left\langle\Psi_{S}^{\mu}\left|\widehat{H}^{\mu}\right| \Psi_{S}^{\mu}\right\rangle$, by using approximate configuration interaction (CI) type wave functions $\Psi_{S}^{\mu}$ expanded into linear combinations of all the few Slater determinants generated from small orbital spaces $S$. The orbitals used are the natural orbitals of the Coulombic system calculated at the MRCISD level. The accuracy of the approximation for $\Psi_{S}^{\mu}$ can be assessed by looking at the difference between $E_{S}^{\mu}$ and $E^{\mu}$

$$
\Delta E_{S}^{\mu}=E_{S}^{\mu}-E^{\mu}
$$

The differences $\Delta E_{S}^{\mu}$ are plotted as a function of $\mu$ in Figure 2 for the He atom with the orbital spaces $S=1 s, S=1 s 2 s$ and $S=1 s 2 s 2 p$. One sees that, in the Coulombic limit, $\mu \rightarrow \infty$, the reduction of the orbital space leads to important errors in the energy. When $\mu$ is decreased, i.e. when the interaction is reduced, the errors due to limited orbital spaces get smaller and smaller. For instance, at $\mu=1$, using only the single-determinant wave function $\Psi_{1 s}^{\mu}$, leads to an error $\Delta E_{1 s}^{\mu}$ of less than 0.005 Hartree. 


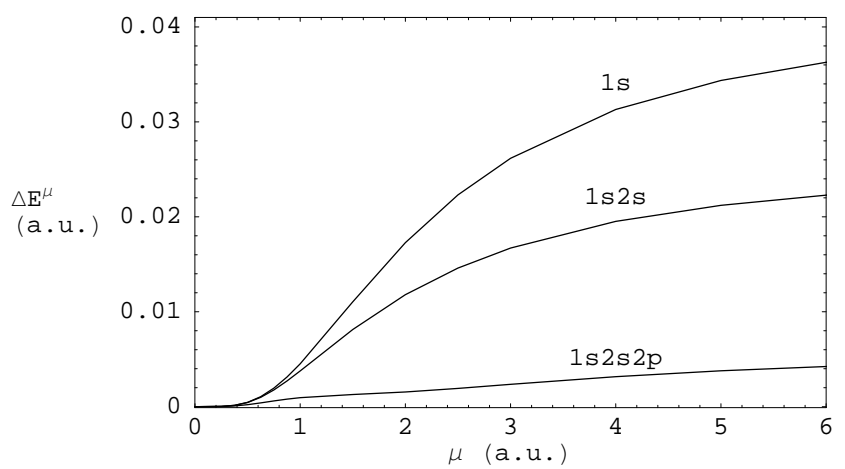

FIGURE 2. Ground-state energy differences $\Delta E_{S}^{\mu}=\left\langle\Psi_{S}^{\mu}\left|\widehat{H}^{\mu}\right| \Psi_{S}^{\mu}\right\rangle-$ $\left\langle\Psi^{\mu}\left|\widehat{H}^{\mu}\right| \Psi^{\mu}\right\rangle$ where $\Psi^{\mu}$ is an accurate wave function and $\Psi_{S}^{\mu}$ are approximate wave functions generated from small orbital spaces $S=1 s, S=1 s 2 s$ and $S=1 s 2 s 2 p$, as a function of $\mu$ for the He atom.

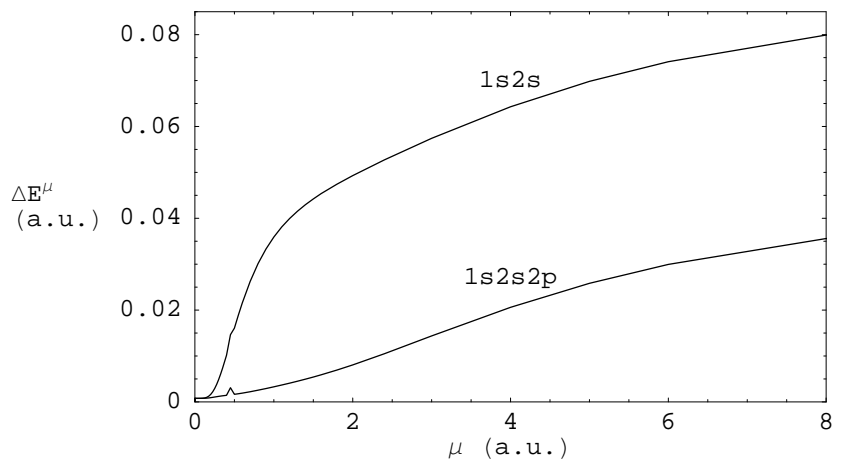

FIGURE 3. Ground-state energy differences $\Delta E_{S}^{\mu}=\left\langle\Psi_{S}^{\mu}\left|\widehat{H}^{\mu}\right| \Psi_{S}^{\mu}\right\rangle-$ $\left\langle\Psi^{\mu}\left|\widehat{H}^{\mu}\right| \Psi^{\mu}\right\rangle$ where $\Psi^{\mu}$ is an accurate wave function and $\Psi_{S}^{\mu}$ are approximate wave functions generated from small orbital spaces $S=1 s 2 s$ and $S=1 s 2 s 2 p$, as a function of $\mu$ for the Be atom.

The case of the Be atom with the orbital spaces $S=1 s 2 s$ and $S=1 s 2 s 2 p$ is reported in Figure 3. Because of the near-degeneracy of the $2 s$ and $2 p$ levels, the inclusion of $2 p$ configurations in the wave function is important, quite independently of the electron-electron interaction. Indeed, the difference $E_{1 s 2 s}^{\mu}-E_{1 s 2 s 2 p}^{\mu}$ remains large for almost all $\mu$ 's. On the contrary, the error of the calculation where the $2 p$ orbitals are included, $\Delta E_{1 s 2 s 2 p}^{\mu}$, quickly falls off when $\mu$ is decreased. Again, for $\mu=1$ for instance, the error $\Delta E_{1 s 2 s 2 p}^{\mu}$ given by the few-determinant CI-type wave function $\Psi_{1 s 2 s 2 p}^{\mu}$ is less than 0.005 Hartree. 
Therefore, the modification of the interaction enables to increase the accuracy of CI-type wave function calculations, or equivalently for a fixed target accuracy, decrease the effort of the calculation by reducing the orbital space. The crucial point for this effect to appear seems to be the reduction of the electron-electron interaction compared to the Coulomb interaction rather than the long-range character of the modified interaction.

\section{Some Recent Results: A Short Overview}

The idea of using the partially interacting model system of (2.1) has been explored with different techniques to compute the wavefunction $\Psi^{\mu}$, and using different approximations for the functionals of Section 3.1. We review very briefly some of the corresponding results.

The LDA functional for $\bar{E}_{\mathrm{xc}}^{\mu}[n]$ has been combined with configuration interaction (CI) to handle the wavefunction $\Psi^{\mu}$ in $[21,27]$; the method has been applied to atoms and small molecules. Again using LDA for the short-range functional, in [1] it has been shown that long-range van der Waals forces in rare gas dimers can be well described by using second-order perturbation theory for $\Psi^{\mu}$.

A short-range GGA functional has been combined with the coupled-cluster (CC) method to describe $\Psi^{\mu}$, with very good results for small molecules, both for the closed and the open shell cases $[9,10]$ : in particular, the results from multideterminantal DFT for small and medium basis-set sizes are better of both the pure DFT result and the pure CC result. In [31], a different GGA functional [16,32] has been used, and $\Psi^{\mu}$ has been determined by multi-configuration self consistent field (MCSCF). The corresponding application to systems in which near-degeneracy effects play a major role is promising, although self-interaction errors in the functionals are still a problem in some cases [31].

Realistic $f^{\mu^{\prime}}\left(r_{12}\right)$ from Eqs. (3.16) have been generated for two-electron atoms in [12]: with a very simple approximation for $w_{\text {eff }}^{\mu^{\prime}}\left(r_{12}\right)$ (inspired to the one used for the electron gas at $\mu=\infty)$, extremely accurate $\bar{E}_{\mathrm{xc}}^{\mu}[n]$ have been obtained. This new strategy of producing functionals is still at a very early stage, and several steps are needed for its development. In particular, we need better approximations for $w_{\text {eff }}^{\mu^{\prime}}\left(r_{12}\right)$, and the implementation of an efficient algorithm to couple the radial equations (3.16) with the method used to solve the model Hamiltonian $\widehat{H}^{\mu}$.

In conclusions, changing the model Hamiltonian in DFT calculations seems to be a promising alternative to the construction of better exchange-correlation functionals for standard Kohn-Sham DFT. Of course, the method has been under development only in the last decade, and it still needs further improvement and investigation in many aspects.

\section{References}

1. J.G. Angyan, I.C. Gerber, A. Savin, and J. Toulouse, van der Waals forces in density functional theory: perturbational long-range electron interaction corrections, Phys. Rev. A 72 (2005), no. 012510, 1-9.

2. K. Burke, J.P. Perdew, and M. Ernzerhof, Why semilocal functionals work: accuracy of the on-top pair density and importance of system averaging, J. Chem. Phys. 109 (1998), 37603771.

3. D. M. Ceperley and B.J. Alder, Ground state of the electron gas by a stochastic method, Phys. Rev. Lett. 45 (1980), 566-569. 
4. A.J. Coleman and V.I. Yukalov, Reduced density matrices: Coulson's challenge, Springer, Berlin, 2000.

5. B. Davoudi, M. Polini, R. Asgari, and M.P. Tosi, Self-consistent Overhauser model for the pair distribution function of an electron gas in dimensionalities $D=3$ and $D=2$, Phys. Rev. B 66 (2002), no. 075110, 1-8.

6. G.E. Engel, and W.E. Pickett, Investigation of density functionals to predict both ground-state properties and band structures, Phys. Rev. B 54 (1996), 8420-8429.

7. W.M.C. Foulkes, L. Mitas, R.J. Needs, and G. Rajagopal, Quantum Monte Carlo simulations of solids, Rev. Modern Physics 73 (2001), 33-83.

8. P.M.W. Gill and R.D. Adamson, A family of attenuated Coulomb operators, Chem. Phys. Lett. 261 (1996), 105-110.

9. E. Goll, H.-J. Werner, and H. Stoll, A short-range gradient-corrected density functional in long-range coupled-cluster calculations for rare gas dimers, Phys. Chem. Chem. Phys. 7 (2005), 3917-3923.

10. E. Goll, H.-J. Werner, H. Stoll, T. Leininger, P. Gori-Giorgi, and A. Savin, A short-range gradient-corrected spin density functional in combination with long-range coupled-cluster methods: application to alkali-metal rare-gas dimer, Chem. Phys. 329 (2006), 276-282.

11. P. Gori-Giorgi and J.P. Perdew, Short-range correlation in the uniform electron gas: extended Overhauser model, Phys. Rev. B 64 (2001), no. 155102, 1-8.

12. P. Gori-Giorgi and A. Savin, Simple model for the spherically- and system-averaged pair density: results for two-electron atoms, Phys. Rev. A 71 (2005), no. 032153, 1-9.

13. System-adapted correlation energy density functionals from effective pair interactions, Phil. Mag. 86 (2006), 2643-2659.

14. _ Properties of short-range and long-range correlation energy density functionals from electron-electron coalescence, Phys. Rev. A 73 (2006), no. 032506, 1-9.

15. C. Gutlé, Espace orbitalaires et théorie de la fonctionnelle de la densité, Ph.D thesis, Université Pierre et Marie Curie, 2003; http://www.lct.jussieu.fr/DFT/ (in french).

16. J. Heyd, G.E. Scuseria, and M. Ernzerhof Hybrid functionals based on a screened Coulomb potential, J. Chem. Phys. 118 (2003), 8207-8215.

17. P. Hohenberg and W. Kohn, Inhomogeneous electron gas, Phys. Rev. B 136 (1964), 864-871.

18. W. Kohn, Nobel lecture: electronic structure of matter - wave functions and density functionals, Rev. Modern Physics 71 (1999), 1253-1266.

19. W. Kohn and L.J. Sham, Self-Consistent equations including exchange and correlation effects, Phys. Rev. A 140 (1965), 1133-1138.

20. S. Kümmel and J.P. Perdew, Optimized effective potential made simple: orbital functionals, orbital shifts, and the exact Kohn-Sham exchange potential, Phys. Rev. B 68 (2003), no. 035103, 1-15; and references therein.

21. T. Leininger, H. Stoll, H.-J. Werner, and A. Savin, Combining long-range configuration interaction with short-range density functionals, Chem. Phys. Lett. 275 (1997), 151-160.

22. M. Levy, Universal variational functionals of electron densities, 1st-order reduced densitymatrices, and natural spin-orbitals and solution of the v-representability problem, Proc. Nat. Acad. Sci. U.S.A. (1979) 76, 6062-6065.

23. E.H. Lieb, Density functionals for Coulomb-systems, Int. J. Quant. Chem. 24 (1983), 243-277.

24. A.E. Mattsson, In pursuit of the "divine" functional, Science 298 (2002), 759-760.

25. S. Paziani, S. Moroni, P. Gori-Giorgi, and G.B. Bachelet, Local-spin-density functional for multideterminant density functional theory, Phys. Rev. B 73 (2006), no. 155111, 1-9.

26. J.P. Perdew, K. Burke, and M. Ernzerhof, Generalized gradient approximation made simple, Phys. Rev. Lett. 77 (1996), 3865-3868.

27. R. Pollet, A. Savin, T. Leininger, and H. Stoll, Combining multideterminantal wave functions with density functionals to handle near-degeneracy in atoms and molecules, J. Chem. Phys. 116 (2002), 1250-1258.

28. J.A. Pople, Nobel lecture: quantum chemical models, Rev. Modern Physics 71 (1999), 12671274.

29. J. Rey and A. Savin, Virtual space level shifting and correlation energies, Int. J. Quant. Chem. 69 (1998), 581-590.

30. A. Savin, F. Colonna, and R. Pollet, Adiabatic connection approach to density functional theory of electronic systems, Int. J. Quant. Chem. 94 (2003), 166-190. 
31. J. Toulouse, Extension multidéterminantale de la méthode de Kohn-Sham en théorie de la fonctionnelle de la densité par décomposition de l'interaction électronique en contributions de longue portée et de courte portée, Ph.D thesis, Université Pierre et Marie Curie, 2005 ; http://www.lct.jussieu.fr/pagesperso/toulouse/ (in french).

32. J. Toulouse, F. Colonna, and A. Savin, Long-range/short-range separation of the electronelectron interaction in density functional theory, Phys. Rev. A 70 (2004), no. 062505, 1-16.

33. _ Short-range exchange and correlation energy density functionals: beyond the local density approximation, J. Chem. Phys. 122 (2005), no. 014110, 1-10.

34. J. Toulouse, P. Gori-Giorgi, and A. Savin, A short-range correlation energy density functional with multi-determinantal reference, Theor. Chem. Acc. 114 (2005), 305-308.

35. J. Toulouse, A. Savin, and H.-J. Flad, Short-range exchange-correlation energy of a uniform electron gas with modified electron-electron interaction, Int. J. Quantum Chem. 100 (2004), 1047-1056.

36. W. Yang, Generalized adiabatic connection in density functional theory, J. Chem. Phys. 109 (1998), 10107-10110.

37. W. Yang and Q. Wu, Direct Method for Optimized Effective Potentials in Density-Functional Theory, Phys. Rev. Lett. 89 (2002), no. 143002, 1-4; and references therein.

Laboratoire de Chimie Théorique, CNRS, Université Pierre et Marie Curie, 4 Place Jussieu, F-75252 PARIs, France.

E-mail address: gori@lct.jussieu.fr

Cornell Theory Center, Frank H.T. Rhodes Hall, Cornell University, Ithaca, New YORK, 14853-3801, USA.

E-mail address: toulouse@cornell.edu

Laboratoire de Chimie Théorique, CNRS, Université Pierre et Marie Curie, 4 Place Jussieu, F-75252 PAris, France

E-mail address: savin@lct.jussieu.fr 